\title{
SWI/SNF-deficient undifferentiated/ rhabdoid carcinoma of the gallbladder carrying a POLE mutation in a 30-year-old woman: a case report
}

Tiemo S. Gerber ${ }^{1}$, Abbas Agaimy ${ }^{2}$, Arndt Hartmann², Michael Habekost ${ }^{3}$, Wilfried Roth ${ }^{1}$, Albrecht Stenzinger ${ }^{4}$, Peter Schirmacher ${ }^{4}$ and Beate K. Straub ${ }^{1 *}$

\begin{abstract}
Background: Undifferentiated carcinoma of the biliary tract are highly aggressive malignancies. In other organs, a subgroup of undifferentiated carcinoma related to SWI/SNF complex-deficiency have been described.

Case presentation: A 30-year-old woman presented with rising inflammatory markers (C-reactive protein (CRP)). Ultrasound examination revealed a large tumor of the liver. A computed tomography scan was performed and was primarily interpreted as a tumor-forming liver abscess, possibly caused by gallbladder perforation. Subsequent liver segment resection was performed. Microscopic examination showed an undifferentiated carcinoma with rhabdoid morphology and prominent inflammatory infiltrate in the gallbladder base. With SWI/SNF immunohistochemistry, intact expression of SMARCB1, SMARCA4, ARID1A, but loss of SMARCA2 and PBRM1 was detected. Next-generationsequencing detected KRAS, PBRM1 and ARID1B mutations, a deleterious splice-site mutation in the POLE-gene and a mutation in the TP53-gene.

Conclusions: We were able to demonstrate loss of SMARCA2 expression and mutations characteristic of an SWI/ SNF-deficient carcinoma in a tumor derived from the gallbladder. This is the first reported case of an undifferentiated carcinoma with rhabdoid features in the gallbladder carrying a POLE mutation and SWI/SNFdeficiency of PBRM1 and SMARCA2.
\end{abstract}

Keywords: Case report, SWI/SNF-deficiency, Gallbladder carcinoma, SMARCA4, Rhabdoid

\section{Background}

Undifferentiated carcinomas (UC) of the gallbladder are malignant neoplasms with poor prognosis, accounting for $3.4 \%$ of all gallbladder carcinomas (GBC) with a median survival of 7.3 months and a mean age of the patients of 67 years. UC most often presents as a polypoid mass of a median size of $5 \mathrm{~cm}$ in radiologic and

\footnotetext{
* Correspondence: beate.straub@unimedizin-mainz.de

'Institute of Pathology, University Medical Center Mainz, Langenbeckstraße 1, 55131 Mainz, Germany

Full list of author information is available at the end of the article
}

pathologic gross examination. The morphology of UC may be variable, varying from small cell type, pleomorphic cell type, to pseudosarcomatous type. [1]

Neoplasms of the gastrointestinal tract with rhabdoid features are distinctive, highly aggressive tumors defined by rhabdomyoblast-like morphology but without evidence for rhabdomyosarcomatous differentiation. Most patients diagnosed with such tumors die within one year, with a mean survival of 4 months. [2, 3]

SNF (sucrose nonfermenting) and SWI (switch) genes encode subunits of large protein complexes that exert a 
crucial role as global regulators of transcription through ATP-dependent chromatin remodeling. SWI/SNF complexes contain $>20$ genes including SMARCA2, SMAR CA4, SMARCC1, SMARCC2, SMARCB1, ARID1A, PBRM1 among others. SWI/SNF complexes play critical roles in cancer cell differentiation and induce aberrant activation of Hedgehog signaling as well as alterations in cellular adhesion and motility. [4] SMARCB1 (INI1, SNF5/BAF47) was the first member of the SWI/SNFcomplex found to be mutated in cancer: families with heterozygous germline SMARCB1 mutations were described with extrarenal rhabdoid tumors with loss of expression of SMARCB1 presenting already in early childhood. [5] Apart from rhabdoid tumors, meanwhile, also in carcinomas, aberrations of SWI/SNF complex proteins have been frequently observed. Commonly mutated SWI/SNF subunits include SMARCA4 inactivation in undifferentiated thoracic malignancies and small cell carcinoma of the ovary, hypercalcemic type $[6,7]$ as well as poorly differentiated sinonasal carcinoma [8], SMAR $C B 1$ inactivation in epithelioid sarcoma [9, 10], PBRM1 truncating mutations in clear cell renal cell carcinoma [11], and inactivating mutations of members of the ARID family in many different cancer types, including cholangiocarcinoma [12]. In dedifferentiated and undifferentiated endometrial carcinoma, $18-66 \%$ of tumors show defective alterations of SWI/SNF complex proteins, of which patients with $P O L E$-mutated cancers demonstrate better prognosis [13-15]. ARID1A and ARID1B, member of the SWI/SNF complexes, are involved in the regulation of transcription, DNA repair, and the epigenetic landscape via the DNA polymerase POLE $[16,17]$. The genetic alterations may be somatic or be linked with germline mutations, resulting in the familial clustering of these tumors. [18] Concerning gastrointestinal undifferentiated carcinoma, loss of SWI/SNF complex proteins has already been described in UC of the stomach, colon, vermiform appendix, duodenum, and the pancreas, often leading to rhabdoid features [19].

We here report a rare case of an undifferentiated carcinoma of the gallbladder in a young woman with unique molecular changes.

\section{Case presentation}

We present the case of a 30-year-old woman with diarrhea and acute onset of upper abdominal pain. She had no personal or family history of cancer. There weren ' $t$ any previous interventions known. Subsequent ultrasound examination revealed a strongly echogenic lesion in liver segment VI. A computed tomographic scan of the abdomen performed with the intravenous administration of contrast medium showed a pericholecystic fluid collection measuring $12 \mathrm{~cm} \mathrm{x} 9 \mathrm{~cm}$ and an irregular thickening of the gallbladder wall, so the presumptive radiological diagnosis of acute cholecystitis complicated by gallbladder perforation and abscess formation was made. Clinical differential diagnoses included liver abscess, cystic echinococcosis, and neoplastic disease, so following tumor board decision, the patient was admitted to open procedure resection with cholecystectomy and liver segment V / VI resection of the lesion.

At gross examination, liver resection showed a tumorforming lesion of $13.5 \mathrm{~cm}$ in size with an irregular, grey surface, with partly crumbling and suppurating, partly indurated texture in close proximity to the gallbladder. The gallbladder was of $6.7 \mathrm{~cm}$ length with a thickened wall of up to $3 \mathrm{~cm}$ and contained yellow concretions. Histological examination revealed a highly pleomorphic, highly proliferative, overtly malignant tumor with hyperchromasia, atypical mitotic figures, eosinophilic cytoplasm, and prominent nucleoli (Fig. 1). Besides, geographic necrosis, multinucleated tumor giant cells and abundant neutrophilic infiltrate were present. The tumor infiltrated the gallbladder wall and adjacent adipose tissue, as well as the liver. In the proximity of the tumor, the gallbladder epithelium displayed atypia with an increased nuclear/cytoplasmic ratio, hyperchromasia, and loss of nuclear polarity, characteristic of high-grade biliary intraepithelial neoplasia. Focal gland formation was observed, reminiscent of an adenocarcinoma (Fig. 2). Immunohistochemistry of the carcinoma revealed positivity solely for cytokeratin 8 and vimentin. Immunohistochemical stains against TTF-1, HepPar1, PAX8, OCT3/4, CEA, BerEp4, estrogen receptor, cytokeratins 7 and 20, as well as CDX2 and ErbB2 were negative. Ki67-proliferation rate (MIB-1 labeling index) was over $95 \%$. PBRM1- and SMARCA2-expression were lost in the carcinoma but retained in the associated high-grade biliary intraepithelial neoplasia. SMARCA4, SMARCB1 (INI-1), and ARID1A were retained. PDL-1 was strongly expressed in $80 \%$ of tumor cells as well as in some inflammatory cells. Next-generation-sequencing with the Oncomine-Comprehensive Assay v3 detected a KRAS mutation (p.Gly12Asp; allele frequency $29,5 \%$ ), a deleterious splice-site mutation in the POLE-gene (NM 006231:c.4729-1G > A; allele frequency 7,3\%) and a TP53 splice site mutation (allele frequency 17,9\%). Besides, a variant of unknown significance in the SMAR CA4-gene (NM_003072:p.Leu1063Val; allele frequency $14,5 \%$ ) was discovered. In addition, PBRM1 and $A R I D 1 B$ mutations were detected, among others (see Table 1 and Supplemental 1). The mutations were also verified using another NGS panel (Illumina TruSight Tumor 500Panel) using two different tumor areas. A comparative analysis of biliary intraepithelial neoplasia and invasive tumor was not possible due to the spatial proximity and tumor spill-over. The mutational burden was increased (12.53 and 11.01 mutations per megabase) [20]. The 
Table 1 Mutations detected with Illumina TruSight Tumor 500 panel. Concerning all mutations as well as copy number alterations see Supplemental Table 1

\begin{tabular}{lllcl}
\hline Gene & Mutation & Location $\mathbf{1}$ & Location 2 & Variant cDNA \\
\hline KRAS & p.Gly12Asp & $\mathbf{2 4 . 8} \%$ & $\mathbf{6 1 . 6} \%$ & c.35G > A \\
TP53 & p.spl? & $\mathbf{1 3 . 7} \%$ & $\mathbf{3 2 . 6} \%$ & c.993 + 1G > T \\
POLE & p.spl? & $\mathbf{4 . 8} \%$ & $\mathbf{1 6 . 5} \%$ & c.4729-1G > A \\
PBRM1 & p.Leu526Val & $\mathbf{2 3 . 9}$ & $\mathbf{4 9 . 1}$ & c.1576T > G \\
ARID1B & p.Ser1869Cys & $/$ & $\mathbf{6 7 . 4}$ & c.5606 C > G \\
\hline
\end{tabular}

tumor was microsatellite stable and showed tumor heterogeneity.

In the postoperative course, elevated serum CRP levels declined under systemic intravenous antibiotics. The patient died within ten months of the diagnosis.

\section{Discussion}

Although two cases of carcinomas of the gallbladder with rhabdoid features have already been described $[21,22]$, this case report describes the first report of undifferentiated carcinoma of the gallbladder with rhabdoid features and SWI/SNF-deficiency. Pathological examination revealed complete dedifferentiation of the tumor, although focal gland formation, retained epithelial marker expression, and high-grade biliary intraepithelial neoplasia helped us to conclude an origin from the gallbladder epithelium. From morphology alone, initial differential diagnostic considerations included e.g. extrarenal rhabdoid tumor, yet loss of expression of SMARCB1 (INI1) was not observed. Another important differential diagnosis was sarcoma not otherwise specified. Biliary intraepithelial neoplasia and focal glandular formation favored the diagnosis of an epithelial origin. Rhabdoid carcinomas may occur in different anatomical locations including the gastrointestinal tract, but they share a similar molecular origin, which results in SWI/SNF-deficiency [3, 23]. Typically, SWI/SNF-deficiency may be restricted to the undifferentiated part of the carcinoma. In a thorough review of the literature, Agaimy and coauthors [2] revealed a total of 39 carcinomas of the digestive tract with similar rhabdoid features in the stomach, colon, small bowel, and distal esophagus. $64 \%$ of patients were 60 years of age or older, which is in contrast to our case of a young, 30-year-old women.

Carcinoma of the gallbladder is a rare biliary tract malignancy in western countries with substantial geographic distribution variation. The development of carcinoma of the gallbladder is associated with chronic inflammation of the gallbladder, dietary factors, and female gender. This malignancy is a multifactorial disorder involving multiple genetic alterations, most commonly KRAS-, TP53- and c-erb$B 2$-genes. [24] Within our case, besides SWI/SNFdeficiency, mutations in the KRAS-, the POLE-gene, and the TP53-gene were detected, but no aberration in c-erb-B2. Mutations in POLE, which encodes proofreading DNA Polymerase, are often associated with high mutational burden, thus leading to an ultramutated phenotype [25]. However, causality for tumor ultramutation has only been described for exonuclease domain mutations (EDM). Pathogenic POLE EDM typically display characteristic genomic alterations and are therefore proposed as a distinct clinical entity. [26]. In our case, the role of the POLE mutation and its pathogenicity remains unclear. The young age of the patient may point to a germline predisposition mutation, but such analyses have not been performed. The mutation in TP53 we

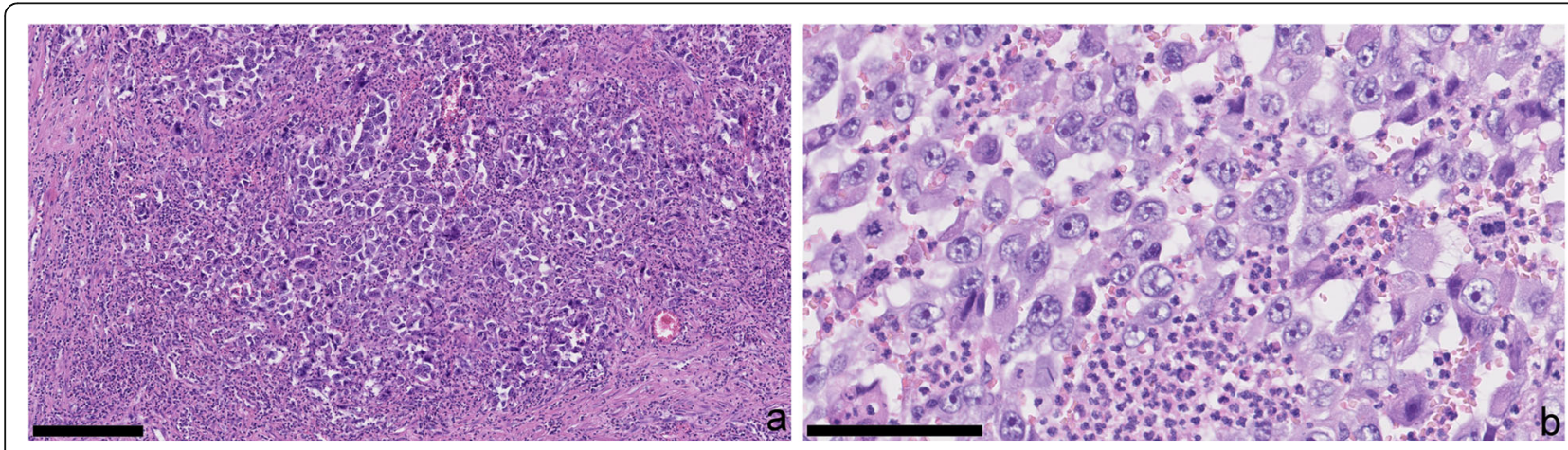

Fig. 1 Histopathology of the undifferentiated carcinoma with rhabdoid features.(a) Morphology shows a highly pleomorphic, highly proliferative carcinoma with, eosinophilic cytoplasm, hyperchromatic nuclei and prominent nucleoli as well as admixed abundant neutrophilic granulocytes. H\&E staining; bar: $250 \mu \mathrm{m}$. (b) high power aspect of the carcinoma (H\&E staining; bar: $100 \mu \mathrm{m}$ ) 


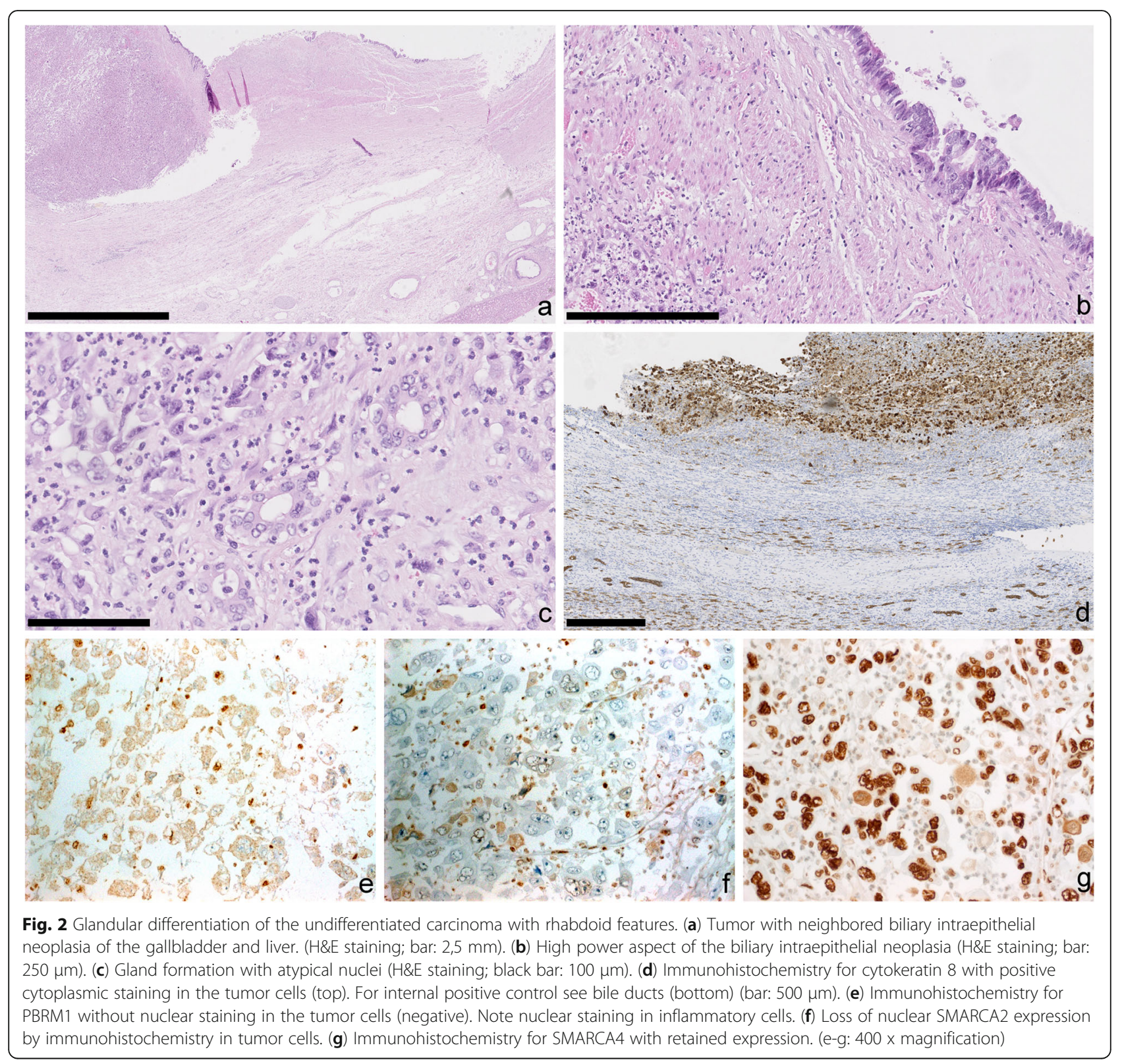

detected in the tumor has been described to be often present as germline mutation. Normal tissue has not been analysed for ethical reasons. The polymerase proofreading-associated polyposis syndrome (PPAP) with missense mutations in the POLE- or POLD1gene was recently described as cause of a new polyposis syndrome with development of colorectal cancers [27]. However, other tumor types like endometrial carcinoma, gastric cancer, breast cancer, melanomas and glioblastomas were also described to occur in this syndrome [28]. In our case, the prominent inflammatory infiltrates together with strong PDL1-expression may open the possibility for therapy with checkpoint inhibitors.

\section{Conclusions}

We here present the unique case of an undifferentiated rhabdoid carcinoma of the gallbladder with POLE gene mutation and SWI/SNF-deficiency. The diagnosis was made based on a precursor lesion of the gallbladder epithelium and the presence of residual glandular tumor structures as well as a loss of SMARCA2 and mutations in PBRM1 and ARID1B. 
We hope that the case demonstrated here will be useful to deepen the understanding of SWI/SNF-deficient carcinomas of the gastrointestinal tract.

\section{Abbreviations}

EDM: Exonuclease domain mutations; GBC: Gallbladder carcinoma; UC: Undifferentiated carcinoma; SWI/SNF: SWItch Sucrose Non-Fermentable; PPAP: Polymerase proofreading-associated polyposis syndrome

\section{Supplementary Information}

The online version contains supplementary material available at https://doi. org/10.1186/s13000-021-01112-4

Additional file 1.

\section{Acknowledgements}

We acknowledge Gunhild Mechtersheimer, Institute of Pathology, Heidelberg for her valuable discussions.

\section{Authors' contributions}

TG and BS wrote the manuscript. WR reviewed the case. PS, AA, AS and AH performed the molecular pathological analysis, contributed to the diagnosis and made useful comments. All authors have read and approved the final version of the manuscript.

\section{Funding}

No funding was received for this study. TG is supported by the Clinician Scientist Fellowship "Else Kröner Research College: 2018_Kolleg.05". Open Access funding enabled and organized by Projekt DEAL.

\section{Availability of data and materials}

Data sharing does not apply to this article as no datasets were generated or analyzed during the current study.

\section{Declarations}

Ethics approval and consent to participate

Not applicable. The information is anonymous, and the individual is deceased.

\section{Consent for publication}

Not applicable.

\section{Competing interests}

The authors declare that they have no competing interests.

\section{Author details}

'Institute of Pathology, University Medical Center Mainz, Langenbeckstraße 1, 55131 Mainz, Germany. Institute of Pathology, Erlangen University Hospital, Erlangen, Germany. ${ }^{3}$ Department of General- and Visceral Surgery, Agaplesion Markus Krankenhaus, Frankfurt am Main, Germany. ${ }^{4}$ Institute of Pathology, University Hospital Heidelberg, Heidelberg, Germany.

Received: 23 December 2020 Accepted: 28 May 2021

Published online: 12 June 2021

\section{References}

1. Park HJ, Jang KT, Choi DW, Heo JS, Choi SH. Clinicopathologic analysis of undifferentiated carcinoma of the gallbladder. J Hepatobiliary Pancreat Sci. 2014;21(1):58-63.

2. Agaimy A, Rau TT, Hartmann A, Stoehr R. SMARCB1 (INI1)-negative rhabdoid carcinomas of the gastrointestinal tract: clinicopathologic and molecular study of a highly aggressive variant with literature review. Am J Surg Pathol. 2014:38(7):910-20.

3. Agaimy A, Daum O, Märkl B, Lichtmannegger I, Michal M, Hartmann A. SWI/ SNF Complex-deficient Undifferentiated/Rhabdoid Carcinomas of the Gastrointestinal Tract: A Series of 13 Cases Highlighting Mutually Exclusive
Loss of SMARCA4 and SMARCA2 and Frequent Co-inactivation of SMARCB1 and SMARCA2. Am J Surg Pathol. 2016:40(4):544-53.

4. Masliah-Planchon J, Bièche I, Guinebretière J-M, Bourdeaut F, Delattre O SWI/SNF Chromatin Remodeling and Human Malignancies. Annu Rev Pathol. 2015;10(1):145-71.

5. Kohashi K, Tanaka Y, Kishimoto H, Yamamoto H, Yamada Y, Taquchi T, Iwamoto Y, Oda Y. Reclassification of rhabdoid tumor and pediatric undifferentiated/unclassified sarcoma with complete loss of SMARCB1/INI1 protein expression: three subtypes of rhabdoid tumor according to their histological features. Mod Pathol. 2016;29(10):1232-42.

6. Le Loarer F, Watson S, Pierron G, de Montpreville VT, Ballet S, Firmin N, Auguste A, Pissaloux D, Boyault S, Paindavoine S, et al. SMARCA4 inactivation defines a group of undifferentiated thoracic malignancies transcriptionally related to BAF-deficient sarcomas. Nat Genet. 2015;47(10):1200-5.

7. Auguste A, Blanc-Durand F, Deloger M, Le Formal A, Bareja R, Wilkes DC, Richon C, Brunn B, Caron O, Devouassoux-Shisheboran M, et al: Small Cell Carcinoma of the Ovary, Hypercalcemic Type (SCCOHT) beyond SMARCA4 Mutations: A Comprehensive Genomic Analysis. Cells 2020, 9(6).

8. Agaimy A, Jain D, Uddin N, Rooper LM, Bishop JA. SMARCA4-deficient Sinonasal Carcinoma: A Series of 10 Cases Expanding the Genetic Spectrum of SWI/SNF-driven Sinonasal Malignancies. Am J Surg Pathol. 2020;44(5):703-10.

9. Richer W, Masliah-Planchon J, Clement N, Jimenez I, Maillot L, Gentien D, Albaud B, Chemlali W, Galant C, Larousserie F, et al. Embryonic signature distinguishes pediatric and adult rhabdoid tumors from other SMARCB1deficient cancers. Oncotarget. 2017:8(21):34245-57.

10. Kohashi K, Yamamoto H, Yamada Y, Kinoshita I, Taguchi T, Iwamoto Y, Oda Y. SWI/SNF Chromatin-remodeling Complex Status in SMARCB1/INI1preserved Epithelioid Sarcoma. Am J Surg Pathol. 2018;42(3):312-8.

11. Varela I, Tarpey P, Raine K, Huang D, Ong CK, Stephens P, Davies H, Jones D, Lin ML, Teague J, et al. Exome sequencing identifies frequent mutation of the SWI/SNF complex gene PBRM1 in renal carcinoma. Nature. 2011; 469(7331):539-42

12. Jiao Y, Pawlik TM, Anders RA, Selaru FM, Streppel MM, Lucas DJ, Niknafs N, Guthrie VB, Maitra A, Argani P, et al. Exome sequencing identifies frequent inactivating mutations in BAP1, ARID1A and PBRM1 in intrahepatic cholangiocarcinomas. Nat Genet. 2013;45(12):1470-3.

13. Tessier-Cloutier B, Coatham M, Carey M, Nelson GS, Hamilton S, Lum A, Soslow RA, Stewart CJ, Postovit LM, Köbel M, et al: SWI/SNF-deficiency defines highly aggressive undifferentiated endometrial carcinoma. J Pathol Clin Res 2020.

14. Espinosa I, Lee C-H, D'Angelo E, Palacios J, Prat J. Undifferentiated and Dedifferentiated Endometrial Carcinomas With POLE Exonuclease Domain Mutations Have a Favorable Prognosis. Am J Surg Pathol. 2017;41(8):1121-8.

15. Stewart CJ, Crook ML. SWI/SNF complex deficiency and mismatch repair protein expression in undifferentiated and dedifferentiated endometrial carcinoma. Pathology. 2015:47(5):439-45.

16. Kurz L, Miklyaeva A, Skowron MA, Overbeck N, Poschmann G, Becker T, Eul K, Kurz T, Schönberger S, Calaminus G, et al: ARID1A Regulates Transcription and the Epigenetic Landscape via POLE and DMAP1 while ARID1A Deficiency or Pharmacological Inhibition Sensitizes Germ Cell Tumor Cells to ATR Inhibition. Cancers (Basel) 2020, 12(4)

17. Tordella L, Khan S, Hohmeyer A, Banito A, Klotz S, Raguz S, Martin N, Dhamarlingam G, Carroll T, González Meljem JM, et al. SWI/SNF regulates a transcriptional program that induces senescence to prevent liver cancer. Genes Dev. 2016:30(19):2187-98.

18. Agaimy A, Foulkes WD. Hereditary SWI/SNF complex deficiency syndromes. Semin Diagn Pathol. 2018;35(3):193-8

19. Tessier-Cloutier B, Schaeffer DF, Bacani J, Marginean CE, Kalloger S, Köbel M, Lee $\mathrm{CH}$. Loss of switch/sucrose non-fermenting complex protein expression in undifferentiated gastrointestinal and pancreatic carcinomas. Histopathology. 2020;77(1):46-54.

20. Campbell BB, Light N, Fabrizio D, Zatzman M, Fuligni F, de Borja R, Davidson S, Edwards M, Elvin JA, Hodel KP, et al. Comprehensive Analysis of Hypermutation in Human Cancer. Cell. 2017;171(5):1042-56.e1010

21. Kim MJ, Yu E, Ro JY. Sarcomatoid carcinoma of the gallbladder with a rhabdoid tumor component. Arch Pathol Lab Med. 2003;127(10):e406-8.

22. Suri VS, Gondal R, Agarwal A, Malhotra V, Sakhuja P, Singhal S. Primary rhabdoid tumor of the gallbladder: report of a case. Surg Today. 2003;33(6):464-6.

23. Biegel JA. Molecular genetics of atypical teratoid/rhabdoid tumor. Neurosurg Focus. 2006;20(1):E11. 
24. Sharma A, Sharma KL, Gupta A, Yadav A, Kumar A. Gallbladder cancer epidemiology, pathogenesis and molecular genetics: Recent update. World J Gastroenterol. 2017;23(22):3978-98.

25. Castellucci E, He T, Goldstein DY, Halmos B, Chuy J. DNA Polymerase $\varepsilon$ Deficiency Leading to an Ultramutator Phenotype: A Novel Clinically Relevant Entity. Oncologist. 2017:22(5):497-502.

26. León-Castillo A, Britton H, McConechy MK, McAlpine JN, Nout R, Kommoss S, Brucker SY, Carlson JW, Epstein E, Rau TT, et al. Interpretation of somatic POLE mutations in endometrial carcinoma. J Pathol. 2020;250(3):323-35.

27. Spier I, Holzapfel S, Altmüller J, Zhao B, Horpaopan S, Vogt S, Chen S, Morak $M$, Raeder $S$, Kayser K, et al. Frequency and phenotypic spectrum of germline mutations in POLE and seven other polymerase genes in 266 patients with colorectal adenomas and carcinomas. Int J Cancer. 2015; 137(2):320-31

28. Mur P, García-Mulero S, del Valle J, Magraner-Pardo L, Vidal A, Pineda M, Cinnirella G, Martín-Ramos E, Pons T, López-Doriga A, et al. Role of POLE and POLD1 in familial cancer. Genet Sci. 2020;22(12):2089-100.

\section{Publisher's Note}

Springer Nature remains neutral with regard to jurisdictional claims in published maps and institutional affiliations.

Ready to submit your research? Choose BMC and benefit from:

- fast, convenient online submission

- thorough peer review by experienced researchers in your field

- rapid publication on acceptance

- support for research data, including large and complex data types

- gold Open Access which fosters wider collaboration and increased citations

- maximum visibility for your research: over $100 \mathrm{M}$ website views per year

At BMC, research is always in progress.

Learn more biomedcentral.com/submissions 